\title{
A New Lossless Image Compression Algorithm Based on Arithmetic Coding
}

\author{
Bruno Carpentieri \\ Dipartimento di Informatica ed Applicazioni "R. M. Capocelli" \\ Universita' di Salerno \\ 84081 Baronissi (SA), Italy
}

\begin{abstract}
We present a new lossless image compression algorithm based on Arithmetic Coding. Our algorithm selects appropriately, for each pixel position, one of a large number of possible, dynamic, probability distributions, and encodes the current pixel prediction error by using this distribution as the model for the arithmetic encoder. We have experimentally compared our algorithm with Lossless JPEG, that is currently the lossless image compression standard, and also with FELICS and other lossless compression algorithms. Our tests show that the new algorithm outperforms Lossless JPEG and FELICS leading to a compression improvement of about $12 \%$ over Lossless JPEG and $10 \%$ over FELICS.
\end{abstract}

\section{Introduction}

Compression is the coding of data to minimize its representation. The compression of images is motivated by the economic and logistic needs to conserve space in storage media and to save bandwidth in communication. In compressed form data can be stored more compactly and transmitted more rapidly. There are two basic reasons for expecting to be able to compress images. First, the information itself contains redundancies in the form of non uniform distribution of signal values (spatial correlation). Second, the act of digitizing contributes an expansion. The compression process is called lossless compression (also reversible or noiseless coding or redundancy reduction) if the original can be exactly reconstructed from the compressed copy; otherwise it is called lossy compression (also irreversible or fidelity-reducing coding or entropy reduction).

Lossless image compression is required by medical imaging, satellite / aerial imaging, image archiving, preservation of precious art work and documents, or any applications demanding complete image fidelity. The objective of a lossless compressor is the elimination of the statistical redundancy intrinsic to the data.

In this paper we present a new lossless image compression algorithm based on Arithmetic Coding. In Arithmetic Coding a source ensemble is represented by an interval between 0 and $I$ on the real number line. Each symbol of the ensemble narrows this interval. A high probability message narrows the interval less than a low probability message, so that high probability messages contribute fewer bits to the encoded message. As the interval becomes smaller, the number of bits needed to specify the interval itself grows. This simple description of Arithmetic Coding has two major difficulties: shrinking the current interval requires the use of high precision arithmetic, moreover no output is produced until the entire input has been coded. The 
general solution to these problems is to output each leading bit as soon as it is known, and then to double the length of the current interval so that it reflects only the unknown part of the final interval. In Witten, Neal, and Clearly [1987] a mechanism is introduced that prevents the current interval from shrinking too much when the endpoints are close to $1 / 2$ but straddle 1/2. A revised Arithmetic Coder, with implementations targeted to bit, char, and word streams, is presented in Moffat, Neal, and Witten [1995].

Compression algorithms often obtain high compression if their model of the data is appropriate. The function of the model is to predict symbols, i.e. to provide a probability distribution for the next source symbol that has to be coded. Arithmetic coding is the only lossless data compression method known that distinctly separates the coding techniques from the stochastic model for the data to be compressed.

The algorithm we present here, selects appropriately, for each pixel position, one of a large number of possible, dynamic, probability distribution, and encodes the current pixel prediction error by using this distribution as the model for the arithmetic encoder.

In this paper we compare experimentally our algorithm with the current lossless image compression standard (Lossless JPEG), with FELICS and with other lossless compression algorithms. Our tests show that our algorithm outperforms Lossless JPEG and FELICS leading to a compression improvement of about $12 \%$ over Lossless JPEG and 10\% over FELICS. The next two sections introduce Lossless JPEG and FELICS and describe the current effort toward the definition of a new lossless JPEG standard. Section 4 presents our new lossless image compression algorithm. Section 5 shows the experimental results obtained by comparing the compression performances of our algorithm with Lossless JPEG, FELICS, onedimensional arithmetic coding and Gzip. It presents also an experimental comparison with CALIC: the probable future lossless image compression standard. Section 6 presents our conclusions and future research directions.

\section{The Lossless JPEG Standard}

A committee known as JPEG (Joint Photographic Experts Group) was formed under the joint auspices of ISO-IEC / JTC1 / SC2 / WG8 and CCITT SGVIII NIC at the end of 1986 for developing an international standard for the compression and decompression of continuous tone, still frame, monochrome and color images. JPEG includes a lossless compression method called Lossless JPEG. Lossless JPEG is wholly independent of the DCT processing of the other methods. The selection of this method was not the result of rigorous competitive evaluation as was the selection of the DCT-based methods.

JPEG lossless mode does not use DCT, since round-offerrors would prevent a DCT calculation from being lossless. It is a Predictive Coding technique: a predictor combines the values of up to three neighboring samples $(\mathrm{A}, \mathrm{B}, \mathrm{C})$; this prediction is subtracted from the actual sample value and the differenceis entropy encoded.

Two different codecs are specified depending on how the prediction error is encoded (Huffman or Arithmetic coding). A predictor (combining the values of up to three neighboring samples) forms a prediction of each pixel value $X$. This prediction is subtracted from $X$ and the difference is entropy encoded through Huffman or Arithmetic Coding. Due to the number of patents that cover arithmetic coding, the 
Arithmetic Coding version of the lossless JPEG standard is almost never used and the vast majority of the applications that use the JPEG standard format use the Huffiman Coding version. An excellent book about the JPEG standard is Pennebaker and Mitchell [1993]. This book includes the complete text of the ISO JPEG standards DIS 10918-1 and draft DIS 10918-2 (the official JPEG specifications).

\section{FELICS}

FELICS is an acronym for Fast, Efficient Lossless Image Compression System. This technique was presented in Howard [1993] and Howard and Vitter [1993]. It was tested on an extensive set of test images from the Landsat satellite and on other general images and it was reported to provide compression results comparable to the one obtained by the lossless modes of JPEG (arithmetic coding version), with the code running up to five times faster. However it does not perform very well on highly compressible images. In particular it has the limitation of using always at least one bit per pixel, regardless of the redundancy in the target image.

The image is visited in raster-scan order, therefore each pixel has two nearest neighbors that have already been coded and whose values are known to both the encoder and the decoder. Except along the top and left edges, these are the pixel above and the pixel to the left of the new pixel. If pixel $\mathrm{P}$, with neighbors $\mathrm{P}_{1}$ and $\mathrm{P}_{2}$, has to be encoded, we call $L$ the minimum between the intensity values of $\mathrm{P}_{1}$ and $\mathrm{P}_{2}, H$ the maximum and $\Delta$ the difference $H-L . \Delta$ is the prediction context of $\mathrm{P}$, used for code parameter selection. There are three possible cases: either the value of $P$ lies between $H$ and $L$, or it lies above $H$, or below $L$. The coding algorithm uses one bit to indicate whether the value of $\mathrm{P}$ lies between $H$ and $L$, an additional bit (when necessary) to indicate whether it is above or below the interval $[L, H]$ and a simple prefix code or a Golomb-Rice code to specify the exact value of $P$.

This coding method is very fast: it uses only single bits and simple prefix codes. FELICS makes an interesting assumption about the image's intensity distribution, assuming that the value of $\mathrm{P}$ lies in the range $[L, H]$ almost half of the times. When the value of $P$ lies outside the central region it is assumed that the above/below situations are symmetrical and the probability decreases with the distance to the range $[L, H]$.

\subsection{Toward a New Lossless JPEG Standard}

The JPEG international standards effort, under the auspices of ISO / IEC Joint Technical Committee, is currently revising the lossless image compression standard. In March 1994 the international standards Working Group 1 of the Standard Committee 29 of ISO / IEC JCT 1 issued a Call for contribution for project 1.29.12, titled "Next Generation Lossless Compression of Continuous-tone Still Pictures". The goals are to provide two variants for the standard. One emphasizing low complexity solutions and one aiming to achieve the highest possible compression (the first probably using Huffman coding as a back end and the second using Arithmetic coding). The Working Group 1 is also working toward the definition of a near-lossless standard.

The Call for Contribution resulted in algorithms from eight organizations: Ricoh, Mitsubishi, University of California at Santa Cruz, NEC, Canon, WMS, HewlettPackard, and Kodak. The WMS contribution is from three individuals affiliated with three universities (X. Wu of the University of Western Ontario, N. Memon of 
Northern Illinois University and K. Sayood of the University of Nebraska). The proposals were evaluated in a first and in a second JPEG lossless WG1 meetings (Epernay, France, July 1995, and Dallas, Texas, U.S.A., November 1995). CALIC, the WMS contribution did best overall on the lossless tests, while ALCM, the UCSC contribution performed well for near-lossless compression, and LOCO I, the HP contribution, showed good performances for low complexity lossless compression. The evaluation process is not yet complete and it is undergoing through periodical meetings of the JPEG lossless WG1.

\section{A New Lossless Image Compression Algorithm Based on Arithmetic Coding}

In this section we present a new lossless image compression algorithm based on arithmetic coding. The goal of this algorithm is to combine the efficiency of arithmetic coding with fast lossless image coding. Our compression algorithm examines the image in raster-scan order and performs repeatedly the following three steps:

- Prediction of the current pixel value and computation of the prediction error. We use a simple and fast two-points predictor.

- Error modeling. Precise characterization of the errors inherent in the prediction process gives us a model that can be used effectively by the arithmetic coder. Our coder models the prediction error through the usage of multiple, dynamic, prediction contexts.

- Prediction error coding (through arithmetic coding). The arithmetic encoder that we implemented is based on the work published in Witten, Neal, and Clearly [1987] and in Moffat, Neal, and Witten [1995], and therefore it should avoid potential patent problems. Arithmetic coding allows us to obtain optimal average code length.

The decoder executes the same steps in reverse order. It receives the, arithmetic coded, prediction error from the encoder, then it models the error distribution to decode the error received and finally it decodes the current pixel by summing the pixel prediction to the prediction error.

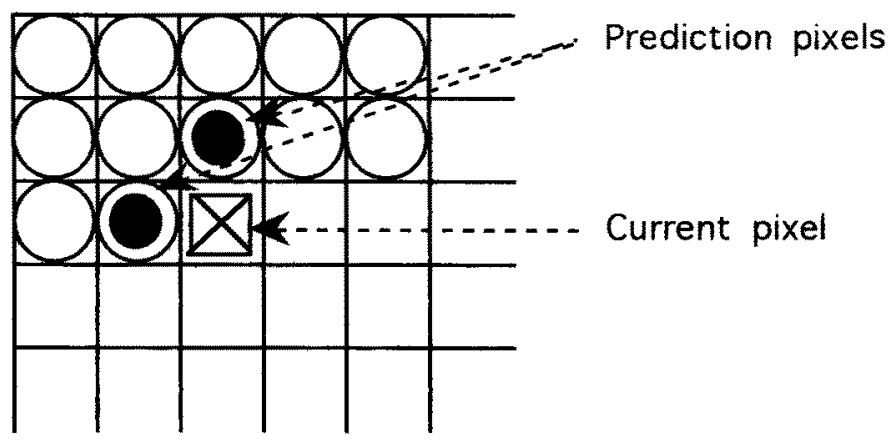

Figure 1: Prediction of the Current Pixel 


\subsection{Prediction of the Current Pixel}

The coder scans the image in raster-scan order and predicts the current pixel as a linear combination of the, already encoded, North and West pixels, as depicted in Figure 1.

After the pixel prediction step, the prediction error $\mathrm{D}_{\mathrm{p}}$ is computed as the difference between the current pixel intensity and the predicted value.

\subsection{Prediction Error Modeling}

A precise characterization of the errors inherent in the prediction process gives us a model that can be used effectively by the arithmetic coder. Our algorithm is a context based method that dynamically identifies the most promising error probability distribution to encode the current pixel prediction error. The algorithm constructs the modeling context for the current error based on the previously computed prediction errors for the neighboring pixels shown in Figure 2. We could use intensity values instead of prediction errors, but in practice using error values works slightly better.

To identify the correct probability distribution we construct a key that is a linear combination of the pixels in the modeling context and we use this key as an index in the probability distributions array. This approach, therefore, leads to a large number of error models that can be addressed easily and rapidly. The probability distributions associated to the contexts are initialized by assigning the same probability to all the possible prediction errors. Successively they are adaptively modified on a context by context basis, in terms of the frequencies of the prediction errors for that context.

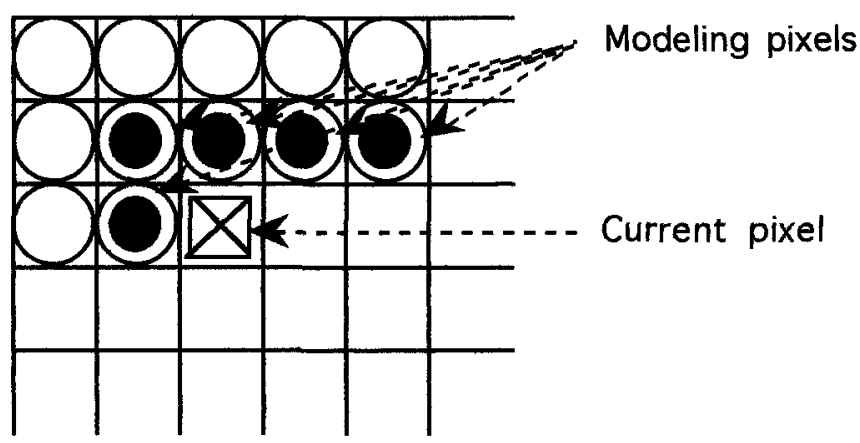

Figure 2: Prediction Error Modeling

\subsection{Prediction Error Coding}

The encoding of the prediction error is performed through arithmetic coding, the input distribution is the one determined by the prediction error modeling step. The arithmetic encoder we have implemented derives from the code developed by Carpinelli, Salamonsen, Moffat, Neal and Witten (see Moffat, Neal, and Witten [1995]). It is a bit-based model that takes one bit at a time from the input stream and passes it and the corresponding context into the statistics module.

\section{Experimental Results}

We have experimentally compared our algorithm with other lossless compression methods. The test data set on which we have tested the compression programs is composed by fifteen images, all 8 bits of grays, specifically: 
- WomanHat - $512 \times 512$, the standard "Lena" image.

- Peppers - 512 x 512, another image widely used in compression experiments.

- Brunette -512 x 512, a first shot of a woman face

- Tank - 512 × 512, a tank that moves on a desolate terrain.

- MilitaryTrucks - $512 \times 512$, two military trucks that moves on a desolate terrain.

- Indian - 512 × 512, a man figure dressed up as an Indian.

- HotelLotus - 512 × 512, another image widely used in compression experiments.

- LivingRoom - 512 × 512, two people in the living room of an old house with light coming in the window.

- LittleGirl - $512 \times 512$, a little girl playing in her room with toys.

- Blonde - 512 x 512, from the standard video sequence "Suzy".

- BrainMR_Side - $256 \times 256$, magnetic resonance medical image that shows a side cross-section of a head.

- ChestCAT - $256 \times 256$, cat-scan chest image.

- BrainMR_Top - 256 × 256, magnetic resonance medical image that shows a top cross-section of a head.

- NASA5 - band 5 of a 7-band image ofDonaldsonville, LA; the least compressible of the 7 bands by UNIX compress.

- NASA6 - band 6 of a 7-band image of Donaldsonville, LA; the most compressible of the 7 bands by UNIX compress.

\begin{tabular}{|lccccc|}
\hline Image & Our & LJPEG & FELICS & Arithmetic & Gzip \\
\hline WomanHat & 1.81 & 1.71 & 1.76 & 1.38 & 1.19 \\
Peppers & 1.72 & 1.65 & 1.67 & 1.36 & 1.15 \\
Brunette & 2.27 & 2.12 & 2.23 & 1.73 & 1.35 \\
Tank & 1.54 & 1.54 & 1.54 & 1.54 & 1.47 \\
MilitaryTrucks & 1.48 & 1.45 & 1.47 & 1.32 & 1.29 \\
Indian & 1.70 & 1.59 & 1.67 & 1.34 & 1.20 \\
HotelLotus & 1.63 & 1.53 & 1.62 & 1.29 & 1.18 \\
LivingRoom & 1.59 & 1.55 & 1.60 & 1.33 & 1.29 \\
LittleGirl & 1.87 & 1.80 & 1.81 & 1.42 & 1.27 \\
Blonde & 1.65 & 1.56 & 1.61 & 1.46 & 1.35 \\
BrainMR_Side & 1.65 & 1.59 & 1.62 & 1.34 & 1.23 \\
ChestCAT & 1.60 & 1.45 & 1.52 & 1.47 & 1.38 \\
BrainMR_Top & 1.27 & 1.25 & 1.24 & 1.10 & 1.08 \\
NASA5 & 1.85 & 1.73 & 1.83 & 1.43 & 1.41 \\
NASA6 & 7.90 & 5.46 & 5.32 & 7.85 & 6.23 \\
\hline
\end{tabular}

Table 1: Experimental Results

In Table 1 we have compared the compression performances of our algorithm with Lossless JPEG and FELICS. The JPEG implementation tested is the public domain Lossless JPEG Codec developed by Kongii Huang and Brian Smith at the Department of Computer Science of the Cornell University. This implementation uses Huffman Coding and not Arithmetic coding (that is patented and not freely available). We have also tested the $m g$-Felics implementation (from the $m g$ system described in Witten, Moffat and Bell [1994]). As mentioned before FELICS is reported to obtain (in average) compression results that are comparable and sometimes better with respect to the arithmetic coding version of Lossless JPEG. As a matter of interest we include 
also both the "bit-oriented" arithmetic program of Moffat, Neal, and Witten [1995] (that is the most efficient on this data set between the algorithms described in that paper) and the UNIX utility Gzip, although they are designed for one-dimensional data and therefore they generally do poorly on images.

In Table 1, for each image we report the compression rates obtained by all these compression algorithms. From the table it can be seen that our algorithm provides an average compression improvement of about $12 \%$ over Lossless JPEG and 10\% over FELICS.

\subsection{An Experimental Comparison with CALIC}

CALIC is a proposal to the Call for Contributions on Lossless Compression of Continuous-tone Still Pictures of ISO / IEC JTC 1, concerning Work Item "Next Generation Lossless Compression / Decompression of Continuous-tone Still Pictures" (the new lossless JPEG standard). This contribution is from three individuals affiliated with three universities (X. Wu of the University of Western Ontario, N. Memon of the University of Northern Illinois and K. Sayood of the University of Nebraska). It was partially presented in Wu [1996] and in Wu [1996b]. CALIC stands for Contextbased, Adaptive Lossless Image Coder; this proposal puts heavy emphasis on image data modeling, it is at the moment the proposal that did best overall on the lossless tests.

CALIC uses a large collection of prediction contexts. it computes two quantities, $d_{h}$ and $d_{v}$, that are estimates, within a given factor, of the gradient magnitude of the intensity function $I[i, j]$ in the horizontal and vertical directions. Using these quantities to detect the orientation and magnitude of the edges, and adjusting the prediction accordingly, CALIC suggests a GAP (Gradient Adjusted) Predictor that weights the neighboring pixels accordingly to the estimate gradients of the image. CALIC context based error modeling has components for local textures and error strength. It uses an innovative context-sensitive error feedback technique: an expected average error, deriving from the past experience with the same context, is added to the prediction. The prediction errors are arithmetic encoded. CALIC is a rather complicated algorithm, its target is compression efficiency. The process that shall lead to a new lossless JPEG standard is still undergoing and therefore CALIC is still subject to changes and modifications. We have tested the (arithmetic coding) implementation of Wu, Wang and Liu that was submitted as a candidate for the new lossless JPEG standard. This implementation was provided as an executable encoder and decoder for a Sun Workstation. We tested it on a Sun SPARCstation under SunOS Release 4.1.3.

The compression results on our test set show that CALIC outperforms our algorithm by an average factor of $14 \%$. For example it gets compression ratio of 1.95 on WomanHat, 2.00 on NASA5, 1.38 on BrainMR_Top. On the other hand the implementation (unoptimized) of our algorithm is faster than CALIC's. On average our encoder runs about $20 \%$ faster than CALIC. This is due to the low complexity of the prediction, modeling, and coding steps with respect to the complicate "tricks" performed by CALIC.

\section{Conclusions and Future Research Directions}

We have presented a new lossless image compression algorithm based on Arithmetic Coding that selects appropriately, for each pixel position, one of a large number of 
possible, dynamic, probability distributions and encodes the current pixel prediction error by using this distribution as the model for the arithmetic encoder. The experimental results show that our algorithm is, in average, more compression efficient with respect to the current Lossless JPEG standard and with respect to FELICS, by a factor of respectively $12 \%$ and $10 \%$. An experimental comparison of our algorithm with CALIC, the probable future lossless compression standard, shows that CALIC achieves better compression (by an average factor of $14 \%$ ) but that it pays this compression improvement by running slower (by an average factor of $20 \%$ ).

Future research directions involve an optimization of the code. We believe that this could lead to a code that runs at least twice as fast. The compression efficiency can be improved by a more accurate prediction step and by including error feedback, although this would lead to a more complicated and slower code.

\section{Acknowledgments}

We thank our student Giovanni Sorrentino for conducting the experimental tests.

\section{References}

P. G. Howard [1993], The design and Analysis of Efficient Lossless Data Compression systems, Ph.D. Dissertation, Department of Computer Science, Brown University, 1993.

P. G. Howard and J. S. Vitter [1993], "Fast and Efficient Lossless Image Compression", Proceedings of the Data Compression Conference 1993, Ed. J. A. Storer and M. Cohn, pp. 351-360, IEEE Press, 1993.

A. Moffat, R. M. Neal, and I. H. Witten [1995], "Arithmetic Coding Revisited", Proceedings of the IEEE, June 1995.

W. B. Pennebaker and J. L. Mitchell [1993], JPEG Still Image Data Compression Standard, Van Nostrand Reinhold, 1993.

M. Weinberger, G. Seroussi and G. Sapiro [1996], "LOCO-I: A Low Complexity, Context -Based, Lossless Image Compression Algorithm", Proceedings of the Data Compression Conference 1996, Ed. J. A. Storer and M. Cohn, pp. 140-149, IEEE Press, 1996.

I. H. Witten, R. M. Neal, and J. M. Clearly [1987], "Arithmetic Coding for Data Compression", Commun. ACM, 30, pp.520-540, June 1987

I. H. Witten, A. Moffat, and T. Bell [1994], Managing Gigabytes, Van Nostran Reinhold, NY, 1994.

X. Wu [1996], "An Algorithmic Study on Lossless Image Compression", Proceedings of the Data Compression Conference 1996, Ed. J. A. Storer and M. Cohn, pp. 150-159, IEEE Press, 1996.

X. Wu [1996b], "Lossless Compression of Continuous-tone Images via Context Selection, Quantization, and Modeling", to appear on IEEE Trans. on Image Processing. 